\title{
Comparative Carbohydrate Catabolism in Corynebacteria
}

\author{
By A. C. ZAGALLO AND C. H. WANG \\ Department of Chemistry and Science Research Institute, \\ Oregon State University, Corvallis, Oregon, U.S.A.
}

(Accepted for publication 7 January 1967)

\begin{abstract}
SUMMARY
The catabolic pathways for the utilization of glucose and gluconate by one strain of each of five species of the genus Corynebacterium were examined. The relative participation of concurrent glucose pathways in each of these five strains was estimated. The findings indicated that Corynebacterium hoagii ATCC 7005 and C. tritici ATCC 11408 displayed catabolic behaviour similar to that of Arthrobacter globiformis ATCC 8010 in that the glycolytic pathway played a major role in glucose catabolism. In contrast, in $C$. equi ATCC 7698 and C. sepedonicum ATCC 9850 , the glycolytic pathway and the pentose cycle pathway played almost equally important roles in glucose utilization. The catabolic pathway functioning in C. xerosis ATCC 7084 was distinct from these other strains of Corynebacteria and resemble that of some Acetobacter strains in that the pentose cycle pathway played a predominant role in the utilization of glucose and gluconate.
\end{abstract}

\section{INTRODUCTION}

The genus Corynebacterium was initially created by Lehman and Neumann (Bergey's Manual, 7th ed., 1957) to contain the diphtheria bacillus and other diphtheria-like organisms parasitic or pathogenic to animals. It has expanded its boundaries ever since, mainly on a morphological basis, to include many more species, from human, other animal, plant and soil sources. As it stands now in the 7th edition of Bergey's Manual (1957), the genus contains organisms of human, animal and plant origin having in common certain features, such as irregularity in shape, which varies from straight, curved, club-shaped or branched rods to almost coccoid organisms. Corynebacteria commonly show the so-called 'snapping division', the organisms show uneven and variable Gram strain and do not have endospores. Some soil organisms formerly in this group have obtained independence and emerged as a new genus Arthrobacter.

The species in the genus Corynebacterium were differentiated primarily on the basis of their habitat, along with some physiological characteristics. However, as stated in a footnote of Bergey's Manual (1957, p. 579), 'habitat relationships are used because comparative studies of the species in these groups are still completely lacking'.

Some studies have been made of the amino acid and carbohydrate composition of the cell wall of a few strains of corynebacteria (Orlova, 1954; Cummins \& Harris, 1955, 1956; Mierzejewski, 1955; Rzucildo, et al, 1958) and on their nutritional characteristics (Lachance, 1960; Keddie, Leask \& Grainger, 1966). Cummins \& Harris (1959), while comparing Arthrobacter with Corynebacteria and other Gram-positive bacteria, 
stressed the importance of cell-wall composition as basis of taxonomy; comparisons of amino acid composition might be of use in separating genera and of varieties of sugars and amino sugars in separating species. Keddie et al. (1966, p. 34) stressed that 'cell-wall composition afford a distinction between saprophytic strains of coryneform bacteria on the one hand and $C$. diphtheriae and related human and animal parasites on the other'.

Only a few strains of Corynebacteria have been investigated in regard to their carbohydrate metabolism. Hulanicka (1960) showed the existence of sedoheptulose and phosphopentose isomerase, epimerase, transketolase and transaldolase in Corynebacterium diphtheriae and concluded the pentose cycle (PC) pathway to be operative in this organism, although the extent of participation of the PC pathway in overall glucose catabolism is not known. The operation of the tricarboxylic acid cycle (TCA) pathway in this organism is reported by Jannes (1953) and Kornberg (1958). Zajic, De Ley \& Starr (1956) reported the operation of the pentose phosphate (PP) pathway and TCA cycle pathway in a strain of $C$. insidiosum, and could not detect a specific dehydrase and aldolase which might convert 6-P-gluconate to pyruvate and triose-P, key enzymes in the Entner-Doudoroff (ED) pathway. Katznelson $(1955,1958)$ was unable to detect the formation of pyruvic acid from glucose, glucose-6-P, fructose-6-P and 6-P-gluconate in C. michiganense, C. fascians and C. flaccumfaciens. In these latter species, the presence of hexokinase, and glyceraldehyde-3-P dehydrogenase was not shown. Evidence for the presence of a poly-phosphate-glucose-phosphotransferase in C. xerosis was reported by Dirheimer \& Ebel (1962).

In view of the lack of a direct comparative study of corynebacteria with respect to carbohydrate metabolism, the present work was undertaken with one strain of each of five species which originated from human, animal and plant sources.

\section{METHODS}

The organisms used were obtained from the American Type Culture Collection.

The strains studied were: Corynebacterium hoagii (ATCC 7005, human); C. equi (ATCC 7698, animal); C. tritici (ATCC 11408, plant); C. sepedonicum (ATCC 9850, plant); C. xerosis (ATCC 7084, human).

The stock cultures were grown on nutrient agar slopes and kept in a refrigerator at about $5^{\circ}$. Subcultures to new media were made at about 3-month intervals. Corynebacterium sepedonicum ATCC 9850 was grown only in liquid medium since its growth on nutrient agar was poor. The medium used for the growth of all Coryne. bacterium strains studied was that used by Lochhead \& Thexton (1951) for the isolation of soil micro-organisms. The main source of carbon was either glucose o1 gluconate, at $0 \cdot 15 \%(\mathrm{w} / \mathrm{v})$. The carbon sources were sterilized separately, by auto. claving $\left(120^{\circ}, 30 \mathrm{~min}\right.$.), as well as some other medium constituents, and mixec aseptically after cooling. The media were adjusted to $\mathrm{pH} 6.8$ with $0 \cdot 1 \mathrm{~N}-\mathrm{HCl}$.

The organisms were grown in $250 \mathrm{ml}$. Erlenmeyer flasks containing about $130 \mathrm{ml}$ liquid medium, and incubated on a rotary shaker at $30^{\circ}$ for Corynebacterium xerosis C. equi and $C$. hoagii, and at $27^{\circ}$ for $C$. tritici and $C$. sepedonicum. Three subculture: from the stock cultures were made at intervals before harvesting the organisms b: centrifugation. The organisms so obtained were immediately resuspended in the growtl medium containing no carbohydrates. Concentrations of organisms in these suspension: 
were determined turbidimetrically, by means of a Klett-Summerson colorimeter, and adjusted to an extinction (640-740 $\mathrm{m} \mu$ ) corresponding to a concentration equivalent to $0 \cdot 7-2 \cdot 7 \mathrm{mg}$. dry weight organism $/ \mathrm{ml}$. Organisms used in experiments were harvested at the late stage of the exponential growth. Organisms grown in glucose medium or in gluconate medium were used.

The labelled substrates were: $\left[1-{ }^{14} \mathrm{C}\right]$ glucose, $\left[2-{ }^{14} \mathrm{C}\right]$ glucose, $\left[3-{ }^{14} \mathrm{C}\right]$ glucose and $\left[6-{ }^{14} \mathrm{C}\right]$ glucose were obtained from the National Bureau of Standards; $\left[1-{ }^{14} \mathrm{C}\right]$ gluconate was bought from the Nuclear Chicago Corporation; $\left[3,4-{ }^{14} \mathrm{C}\right]$ glucose was prepared in this laboratory from liver glycogen of rats metabolizing ${ }^{14} \mathrm{CO}_{2}$ according to the method of Wood, Lifson \& Lorber $(1945)$; $\left[2-{ }^{14} \mathrm{C}\right]$ gluconate, $\left[3-{ }^{14} \mathrm{C}\right]$ gluconate and $\left[6-{ }^{14} \mathrm{C}\right]$ gluconate were prepared from the corresponding labelled glucose samples by the method of Moore \& Link (1940).

The radiorespirometric experiments were done according to the procedures described by Wang (1963). In a typical experiment, $10 \mathrm{ml}$. of a given suspension of organisms were placed in an incubation flask and allowed to utilize a prescribed amount of the labelled substrate under aerobic growth conditions. Respiratory ${ }^{14} \mathrm{CO}_{2}$ released by the organisms was swept through $10 \mathrm{ml}$. of an ethanolamine + ethanol solution $(1+2$, by vol.), which was replaced at hourly intervals. The experiment was terminated when the rate of production of respiratory ${ }^{14} \mathrm{CO}_{2}$ declined to an insignificant value, indicating that the given substrate had been exhausted from the medium. The vessels containing the suspensions were then cooled in ice for about $10 \mathrm{~min}$., after which the organisms were separated by centrifugation. Both the washed organisms and the supernatant fluid thus obtained were assayed for radioactivity.

The radioactivity in respiratory ${ }^{14} \mathrm{CO}_{2}$ was assayed by means of liquid scintillation counting with a Packard Tricarb Spectrometer (Model 314-EX2) in the manner described by Wang (1963). The radioactivity of the organisms and the metabolic products in the media was determined by liquid scintillation counting with the thixotropic gel techniques (White \& Helf, 1956). The efficiency of the liquid scintillation counter for different types of samples was determined by using the internal standards technique. Counts were made over a sufficient period to give a relative standard deviation no greater than $2 \%$.

\section{RESULTS}

All the Corynebacterium strains selected for the present work are capable of utilizing either glucose or gluconate as a sole source carbon. However, except in the case of Corynebacterium sepedonicum ATCC 9850 growth rates were generally better with glucose than with gluconate. Organisms grown in the glucose medium and the gluconate medium were used for the experiments, with ${ }^{14} \mathrm{C}$-labelled glucose or ${ }^{14} \mathrm{C}$-labelled gluconate as substrates. Regardless of the nature of the carbon source in the original culture medium, the radiorespirometric findings for a given substrate were essentially the same. However, with $C$. hoagii ATCC 7005, the utilization of gluconate by glucosegrown organisms was very sluggish, and hence, in this case only gluconate-grown organisms were used with ${ }^{14} \mathrm{C}$ specifically labelled gluconate as substrate.

The results of an experiment on the utilization of ${ }^{14} \mathrm{C}$-labelled glucose or gluconate by the five Corynebacterium strains are given in Table 1 . The data were those observed at the completion of substrate utilization which generally took 6 to $8 \mathrm{hr}$. 
A. C. ZAGALlo AND C. H. WANG

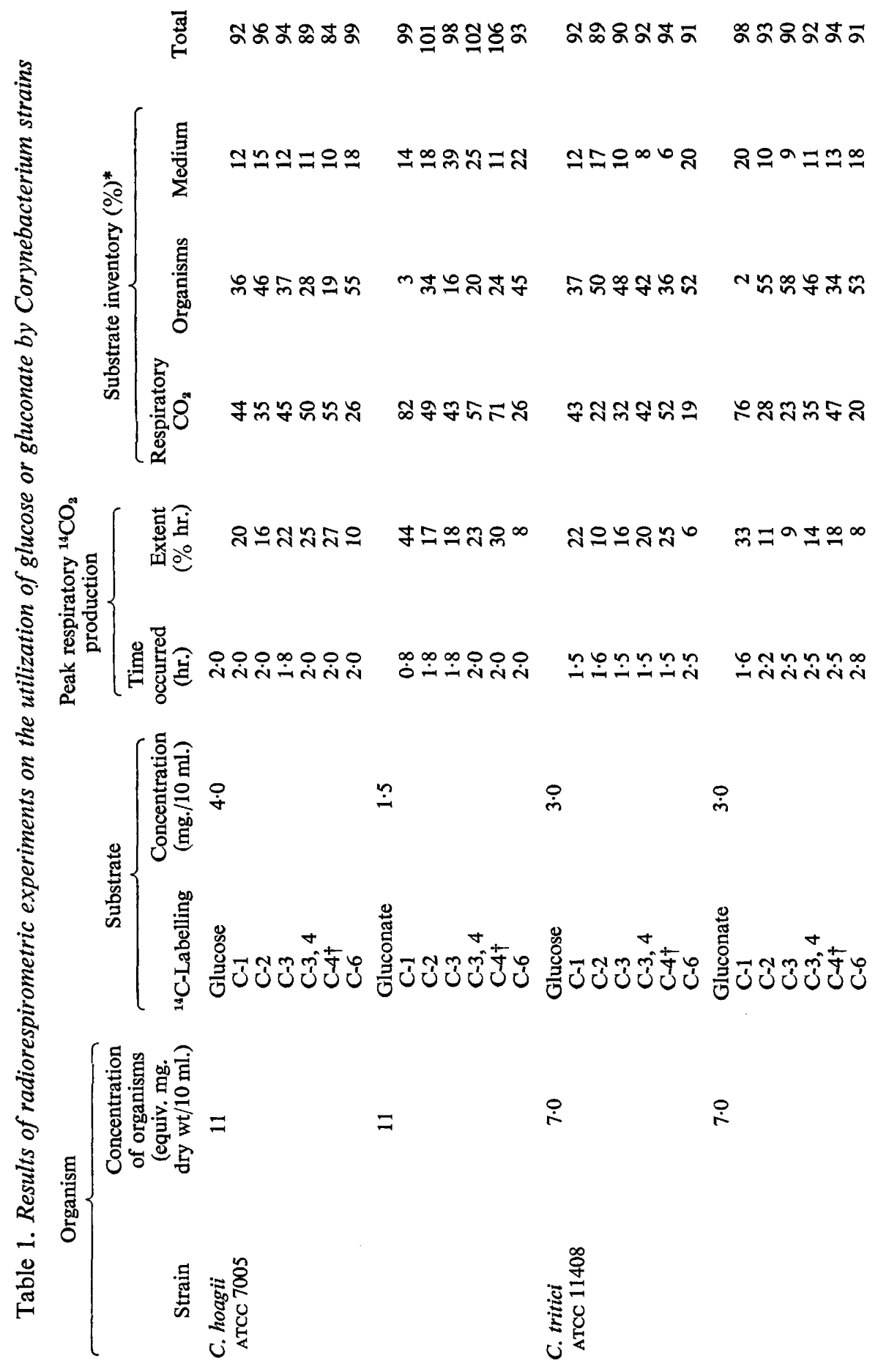




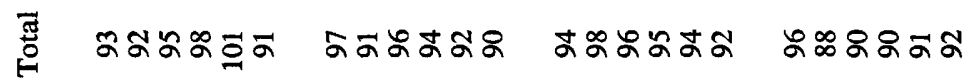

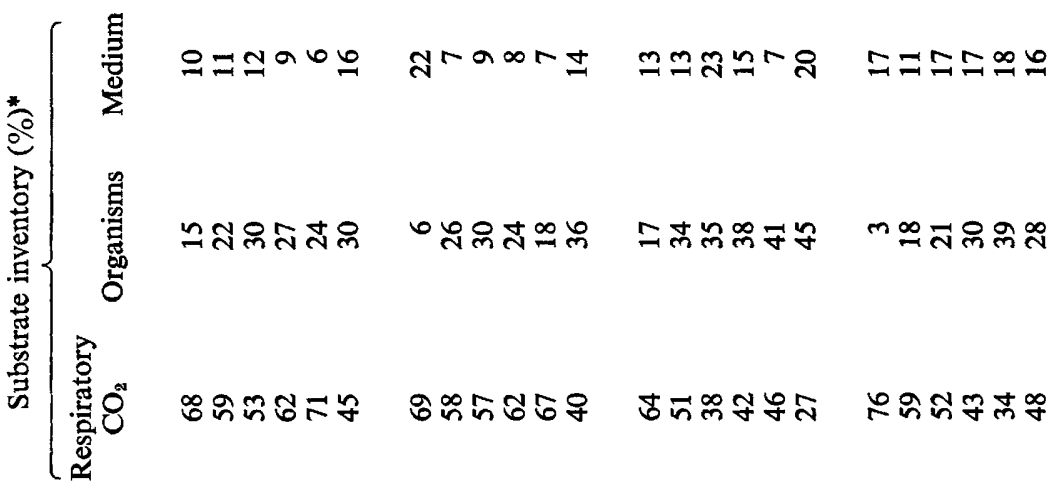
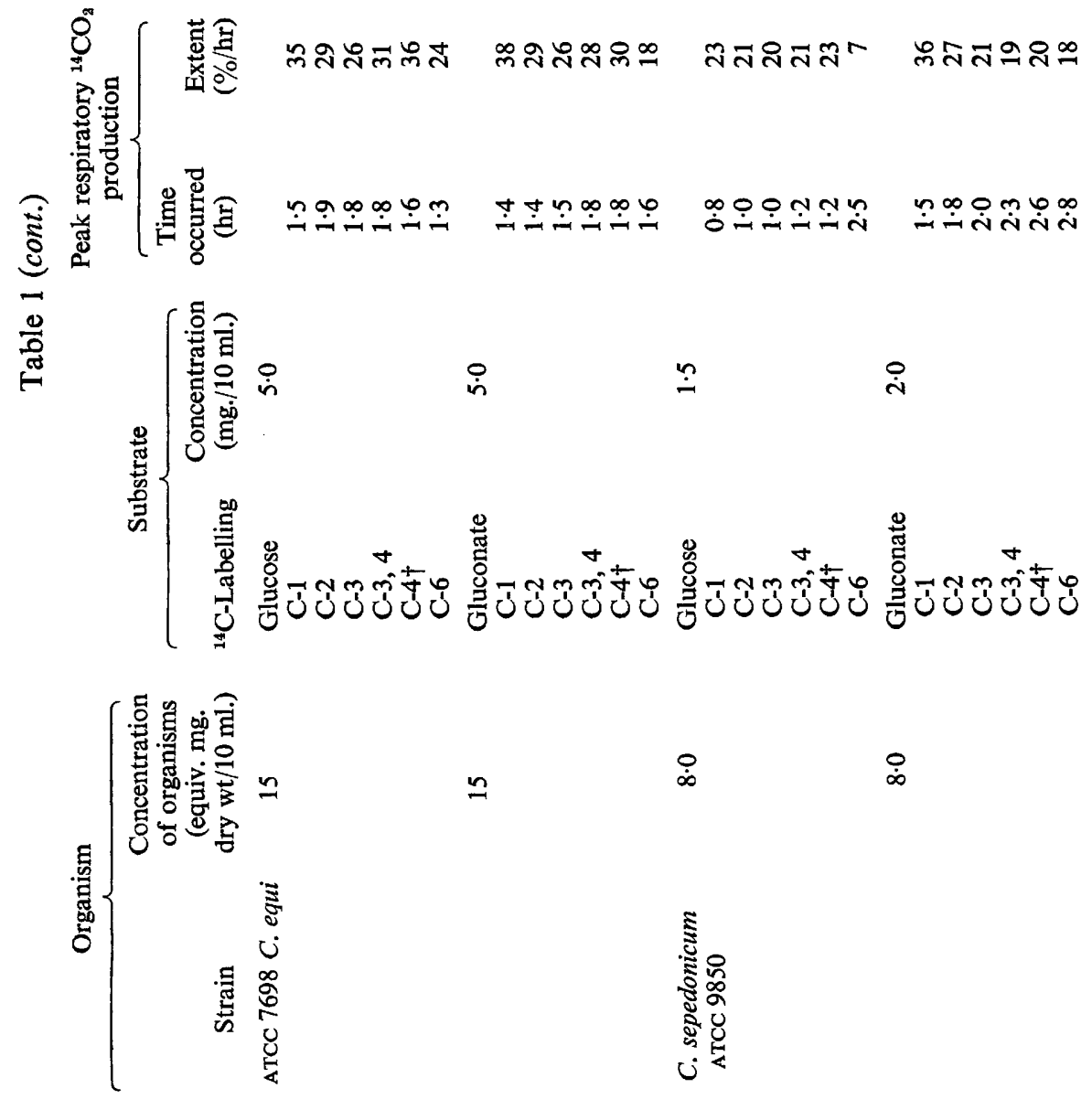


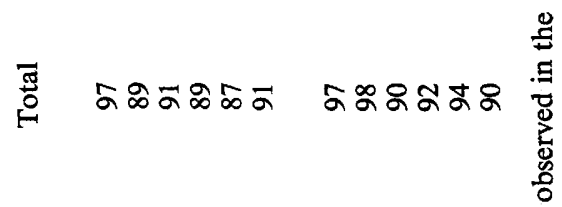

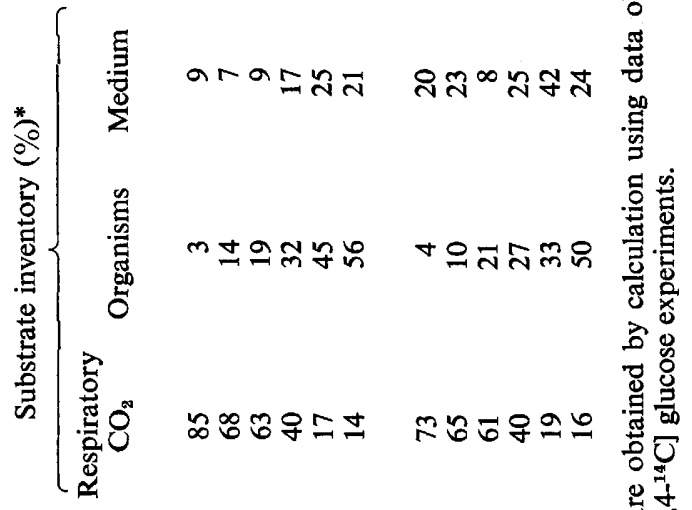

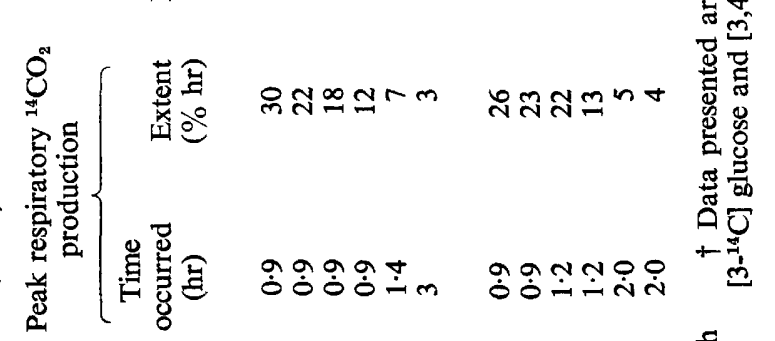

䒕

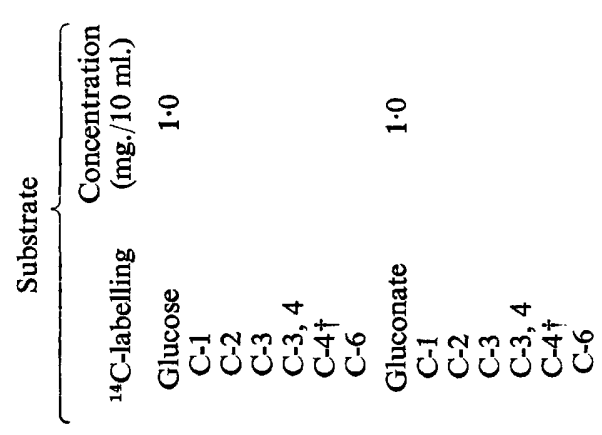

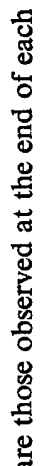

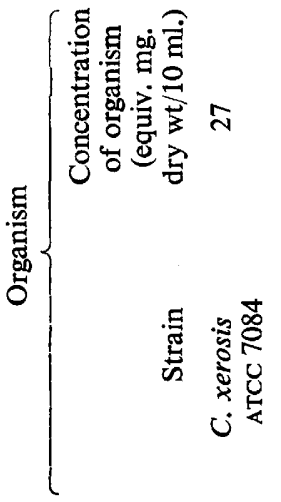

స

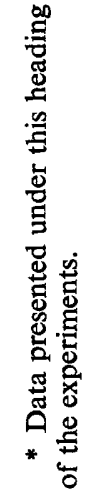


The data in Table 1 represent the average of at least four replicate experiments; the deviation among replicates was less than $5 \%$.

The analysis of the data with regard to the identification and estimation of glucose and gluconate pathways functioning in the various organisms relied on the following considerations. (1) Comparison of the extent of ${ }^{14} \mathrm{CO}_{2}$ production from the various labelled carbon atoms of the substrate at the end of utilization. (2) Comparison of the maximal rates of ${ }^{14} \mathrm{CO}_{2}$ production from labelled carbon atoms of the substrate (see Table 1, column headed 'peak respiratory ${ }^{14} \mathrm{CO}_{2}$ production'). (3) Since gluconate is a key intermediate in several glucose pathways, including the PP, the PC and the ED pathways, much information on the nature of the glucose pathway can be derived by a comparison of the over-all respirometric patterns observed in the glucose and gluconate experiments. Such a comparison assumes that the given gluconate behaved similarly to that derived from glucose catabolism. With this in mind, the information about glucose and gluconate pathways as found in the present work, can be summarized as follows.

Corynebacterium hoagii ATCC 7005. With glucose, the extensive conversion of C-3 and $\mathrm{C}-4$ of glucose to respiratory $\mathrm{CO}_{2}$ led to the conclusion that the EmbdenMeyerhof-Parnas (EMP) pathway played a major role in this organism. The fact that the conversion of $\mathrm{C}-1$ of glucose to $\mathrm{CO}_{2}$ was significantly greater in extent than the conversion of C- 6 provided evidence that the PP pathway was also operative. The operation of the latter pathway was further indicated by the findings in the gluconate experiments, where the conversion of $\mathrm{C}-1$ of gluconate to $\mathrm{CO}_{2}$ was prompt and extensive. However, pentose phosphate derived from the glucose via the PP pathway did not appear to be catabolized via the $\mathrm{PC}$ pathway. This conclusion was drawn from the fact that the extent and rate of conversion of $\mathrm{C}-2$ of gluconate to $\mathrm{CO}_{2}$ was considerably lower than that of the $\mathrm{C}-1$ atoms. In fact, the rate and extent of conversion of gluconate carbon atoms other than $\mathrm{C}-1$ to $\mathrm{CO}_{2}$ appeared to reflect the operation of the sequential catabolic pathway previously described for the organism Arthrobacter ureafaciens ATCC 7562 Arthrobacter globiformis ATCC 8010 (Zagallo \& Wang, 1962) which was given as follows:

$\begin{aligned} \text { gluconate } & \rightarrow \text { 6-phosphogluconate } \rightarrow \text { pentose phosphate } \rightarrow \text { fructose-6-phosphate } \\ \rightarrow & \rightarrow \text { fructose-1, 6-phosphate } \rightarrow \text { triose phosphate } \rightarrow \text { acetyl CoA, etc. } \\ & \longrightarrow \text { - }\end{aligned}$ (* predominantly)

Corynebacterium tritici ATCC 11408. Much of the discussion on the radiorespirometric findings with the $C$. hoagii ATCC 7005 also applies to $C$. tritici ATCC 11408 . Glucose appeared to be metabolized by the $C$. tritici strain primarily via the EMP pathway and to some extent by the PP pathway; gluconate was metabolized via the PP pathway, and the pentose derived from gluconate was incorporated to fructose-6phosphate and subsequently utilized via the EMP pathway or into cellular material instead of being engaged to any great extent in the pentose cycle reactions.

Corynebacterium equi ATCC 7698. The interpretation of the radio-respirometric pattern of the utilization of glucose by this organism was difficult. Superficially it appeared that the ED pathway, shown to function in pseudomonads (Stern, Wang \& Gilmour, 1960), might have been operative in the $C$. equi strain since a close equivalence in catabolic behaviour (rates and extent of conversion of carbon atoms to respiratory 
${ }^{14} \mathrm{CO}_{2}$ ) between $\mathrm{C}-1$ and $\mathrm{C}-4$ of glucose and that between $\mathrm{C}-3$ and $\mathrm{C}-6$ of glucose were indicated by the radiorespirometric data. However, the maximal rate of ${ }^{14} \mathrm{CO}_{2}$ production from the C-6 of glucose occurred significantly earlier than that from the C-3 and the extent of conversion of C-4 of glucose to respiratory $\mathrm{CO}_{2}$ was slightly greater than that of $\mathrm{C}-1$. This led to a closer examination of the findings in the gluconate experiment. With gluconate as substrate, the catabolic equivalences, as indicated by the rates and extents of ${ }^{14} \mathrm{CO}_{2}$ production, between $\mathrm{C}-1$ and $\mathrm{C}-4$ of gluconate were not observed. Instead, the conversion of $\mathrm{C}-2$ and $\mathrm{C}-3$ of gluconate to respiratory ${ }^{14} \mathrm{CO}_{2}$ was only slightly less (in rate and extent) than that of the $\mathrm{C}-1$ of gluconate. Since gluconate is a key intermediate of glucose metabolism in the ED pathway, it can therefore be concluded that in C. equi ATCC 7698 glucose was probably catabolized by way of the EMP glycolytic pathway and the PP pathway. The pentose phosphate derived from either glucose or gluconate via the PP pathway appeared to engage rather extensively in the PC pathway.

Another feature of the catabolic behaviour of C. equi ATCC 7698 was reflected in that the conversion of the C-6 of glucose and, to some extent, the C- 6 of gluconate to respiratory $\mathrm{CO}_{2}$ were extensive in rate and extent. These findings presumably related to the derivation of glyceral-dehyde-3-P, from either the EMP pathway or the PC pathway, being engaged extensively in the sequential operation of the triose-P isomerase and reversed aldolase reactions, leading to the formation of hexose phosphates. Such a mechanism would result in the transference of C-6 of glucose or gluconate to the C-1 position of re-formed hexose phosphates. Further catabolism of the re-formed hexose phosphates, by way of the PP pathway, would give a relatively more direct conversion of the C-6 of glucose or of gluconate to respiratory $\mathrm{CO}_{2}$.

Corynebacterium sepedonicum ATCC 9850 . Glucose appeared to be metabolized by this organism extensively by way of the PC pathway, as shown by the fact that, with glucose as substrate, the conversion of C-2 and C-3 of glucose was only slightly less quick and extensive as compared with that of C-1. Similar results were obtained in the gluconate experiments. Since the conversion of the C-4 of glucose to $\mathrm{CO}_{2}$ was also quick and extensive, the EMP pathway was presumably also playing a role in the over-all catabolism of glucose.

Corynebacterium xerosis ATCC 7084. Both glucose and gluconate appear to be catabolized by this organism extensively, if not exclusively, by way of the PP and PC pathways. This conclusion was supported by the extensive conversion of $\mathrm{C}-2$ and C-3 of glucose or gluconate to $\mathrm{CO}_{2}$, with the extents of conversion only slightly less than that of the C-1 of either substrates. The slow conversion of C-4 and C- 6 of glucose or gluconate to respiratory $\mathrm{CO}_{2}$ indicated that the catabolism of the glyceraldehyde-3-P, derived from the PP and PC pathways, was much limited in extent. However, a significant amount of C-6 of these substrates was incorporated into cellular constituents, a fact which reflected the important role played by glyceraldehyde-3-P in the biosynthetic functions.

On the basis of the foregoing analysis of the data, the relative participation of concurrent primary glucose pathways in the various organisms can be estimated by the method of Wang \& Krackov (1962). The definition of the term 'primary glucose pathway' is that defined by Wang \& Krackov (1962) and by Wang (1964). The estimated values for the relative participation of concurrent pathways are given in Table 2 . The pathway estimations presented in Table 2 represent only a crude assessment of the 
relative participation of the concurrent pathways. This is so because the derivation of the equations for the pathway estimation, when applied to the organisms used in the present work, does not cover complicating factors such as the operation of the triose recombination pathway and the incorporation of intact glucose into cellular constituents, etc. Nevertheless, these complicating factors should not affect greatly the estimated values for participated pathways.

\section{Table 2. Estimated relative participation of concurrent glucose pathways} in five Corynebacterium strains

\begin{tabular}{lccc}
\multicolumn{1}{c}{ Strain } & $\begin{array}{c}\text { Relative participation of } \\
\text { primary glucose pathways } \\
\text { (\% of the given substrate } \\
\text { glucose) }\end{array}$ & Extent of pentose \\
cycle operation & Limited \\
C. hoagii ATCC 7005, human & 78 & 22 & Limited \\
C. tritici ATCC 11608, plant & 70 & 30 & Extensive \\
C. equi ATCC 7698, animal & 62 & 38 & Extensive \\
C. sepedonicum ATCC 9850 , plant & 44 & 56 & Extensive \\
C. xerosis ATCC 7084, human & 15 & 85 &
\end{tabular}

* The relative participation of the PP pathway in over-all glucose catabolism, as estimated by the method of Wang \& Krackov (1962), was calculated in a direct manner and is hence a more reliable piece of information. On the other hand, the relative participation of the EMP pathway was estimated by difference and is subject to inaccuracies due to complicating factors. For $C$. xerosis ATCC 7084, it is possible that the EMP pathway did not play a role in the utilization of glucose.

\section{DISCUSSION}

Despite the classification of the five strains of organisms used in the present study in the genus Corynebacterium, the present findings indicate that there is marked differences in the catabolic mechanism of glucose catabolism between Corynebacterium hoagii ATCC 7005 and C. tritici ATCC 11408. Both organisms use markedly for glucose catabolism, the EMP pathway and to a lesser extent the PP pathway. The pentose phosphate derived from glucose via the PP pathway was not used to any significant extent in the PC relations. Such a catabolic behaviour resembles closely that found with strains of Arthrobacter globiformis ATCC 8010 and A. ureafaciens ATCC 7562. On the other hand, the organisms C. equi ATCC 7698, C. sepedonicum ATCC 9850 and C. xerosis ATCC 7084 displayed a significantly different catabolic behaviour with regard to both glucose and gluconate. In these three strains, the PP pathway played a more important role in glucose catabolism in comparison to the EMP pathway, and the pentose derived from glucose via the PP pathway was engaged in extensive catabolism via the PC pathway. In the case of $C$. xerosis ATCC 7084 the organism used heavily, if not exclusively, the $\mathrm{PC}$ pathway for glucose utilization. Such a catabolic behaviour resembles very much that found with several Acetobacter species (Kitos et al. 1958; Wang \& Bjerre, 1961).

On the basis of the present findings, it appears that the five strains of the genus Corynebacterium studied can be grouped in three categories. The strains $C$. hoagii ATCC 7005 and $C$. tritici ATCC 11408 appear to belong to a category with catabolic behaviour for glucose and gluconate utilization resembling that of some of the 
Arthrobacter strains typified by Arthrobacter globiformis ATCC 8010 (Zagallo \& Wang, 1962). The strains C. equi ATCC 7698 and C. sepedonicum ATCC 9850 tested here resemble each other in that glucose is utilized by a more or less equal contribution of the EMP and PC pathways. The strain C. xerosis ATCC 7084 is distinct in its catabolic behaviour for both glucose and gluconate, with the PC pathway being the predominant route, as in some strains of Acetobacter and Gluconobacter.

The grouping of the Corynebacterium strains examined here by their biochemical behaviour, particularly their catabolic behaviour, does not seem to be related to the sources from which these organisms were initially isolated. Inasmuch as the cell wall compositions of the respective strains included in the present study are not known, it is not possible to correlate the present findings with the taxonomic classifications on cell wall compositions.

This work was supported by a grant from the National Institutes of Health, U.S. Public Health Service (Grant no. AI 01144-11).

\section{REFERENCES}

Bergey's Manual of Determinative Bacteriology, 7th ed. (1957). Ed. by R. S. Breed, E. G. D. Murray and N. R. Smith. Baltimore: The Williams and Wilkins Co.

Cummins, C. S. (1959). Taxonomic position of Arthrobacter. Nature, Lond. 184, 831.

Cummins, C. S. \& HARRIS, H. (1955). Differences in cell-wall composition among gram-positive cocci and bacilli. J. gen. Microbiol. 13, iii, iv.

CUMmins, C. S. \& Harris, H. (1956). A comparison of cell-wall composition in Nocardia, Actinomyces, Mycobacterium and Propionibacterium. J. gen. Microbiol. 15, ix.

DiRheimer, G. \& Ebel, J. P. (1962). Evidence of a polyphosphate-glucose-phosphotransferase in C. xerosis. C. r. hebd. Séanc. Acad. Sci., Paris, 254, 2850.

HULANICKA, D. (1960). The pentose cycle in Corynebacterium diphtheriae. Acta biochim. polon. 7, 449.

JANNES, L. (1953). The tricarboxylic acid cycle in the metabolism of Corynebacterium diphtheriae. Experientia 9, 297.

KATZNELSON, H. (1955). The metabolism of phytopathogenic bacteria. I. Comparative studies on the metabolism of representative species. J. Bact. 70, 469.

KATZNELSON, H. (1958). The metabolism of phytopathogenic bacteria. II. Metabolism of carbohydrates by cell-free extracts. $J$. Bact. 75, 540 .

KFDDIE, R. M., LeASK, B. G. S. \& GraingeR, J. M. (1966). A comparison of coryneform bacteria from soil and herbage: cell wall composition and nutrition. J. appl. Bact. 29, 17.

Kitos, P. A., Wang, C. H., Mohler, B. A., King, T. E. \& Cheldelin, V. H. (1958). Glucose and gluconate dissimilation in Acetobacter suboxydans. J. biol. Chem. 233, 1295.

KORNBERG, H. L. (1958). Metabolism of $C_{2}$ compounds in microorganisms. I. The incorporation of acetate-2- $\mathrm{C}^{14}$ by $P$. fluorescens and by a Corynebacterium, grown on ammonium acetate. Biochem. J. 68, 585 .

LACHANCE, R. A. (1960). The vitamin requirements of Corynebacterium sepedonicum. Can. J. Microbiol. 6, 171 .

LochHeAD, A. G. \& TheXton, R. H. (1951). Qualitative studies of soil microorganisms. X. Bacteria requiring vitamin $B_{12}$ as a growth factor. J. Bact. 63, 219.

MIERZEJEWSKI, T. (1955). Carbohydrate composition of the envelope of Corynebacterium equi.: Annls Univ. Mariae Curie-Sklodowska, 10, 93 (in Polish).

MOORE, S. \& LINK, K P. (1940). Carbohydrate characterization. J. biol Chem.. 133, 293.

OrLova, O. K. (1954) Carbohydrates of the diphtheria bacillus. Biokhimiya 19, 449 (in Russian).

Rzucildo, L., Stachow, A., NowakowsKa, A. \& Kubica, J. (1958). Chemical structure and biological properties of cell walls of Corynebacterium diphtheriae. Bull. Acad. pol. Sci. Cl. II Sér. biol. 6, 21.

Stern, I. J., WANG, C. H. \& Gilmour, C. M. (1960). Comparative catabolism genus Xanthomonas. J. bact. 87, 213.

WANG, C. H. (1963). Metabolism studies by radiorespirometry. Adv. Tracer Methodol. 1, 274.

WANG, C. H. (1964). Comparative glucose catabolism in biological systems. In Taxonomic Biochemistry. Serology. Ed. by C. A. Leone. New York: The Ronald Press. 
WANG, C. H. \& BJERre, S. H. (1961). Comparative carbohydrate catabolism in Acetobacter species. Fed. Proc. 20, 84a.

WANG, C. H. \& Krackov, J. (1962). The catabolic fate of glucose in Bacillus subtilis. J. biol. Chem. 237, 3614.

WhITE, C. G. \& HelF, S. (1956). Suspension counting in scintillation gels. Nucleonics 14, 46.

WOOD, H. G., LIFSON, N. \& LORBER, V. (1945). The position of fixed carbon in glucose from rat liver glycogen. J. biol. Chem. 159, 475.

ZAGALLO, A.C. \& WANG, C. H. (1962). Comparative carbohydrate metabolism in Arthrobacter. J. gen. Microbiol. 29, 389.

Zaje, J. E., De LeY, J. \& STARR, M. P. (1956). Oxidative metabolism of Corynebacterium insidiosum. Bact. Proc. p. 116. 